\title{
An Innovative Smart Grid Framework for Integration and Trading
}

\author{
Fabio Silva ${ }^{1,2, *}$, and Brian O’Regan ${ }^{1,2}$ \\ ${ }^{1}$ International Energy Research Centre, Tyndall National Institute, University College Cork (UCC), Cork, Ireland \\ ${ }^{2}$ IEEE Power \& Energy Society Member
}

\begin{abstract}
Smart Grids (SGs) are at the forefront of the renewable resources transformative change for power generation. Due to its decentralised energy generation approach and potential reduction of the cost of power, its relevance for the energy sector is insurmountable. However, new business models and processes are necessary, and they come with integration, implementation, and operation-specific challenges. This work offers a broad analysis of SG' main architectural aspects concerning security issues, integration bottlenecks and standardisation shortcomings in the development of an efficient platform for local energy (generation and storage) surplus trading. Through a multi-layered smart grid architecture description, this work develops an in-depth depiction of the interoperability between these layers (from top business layer passing through information and communications layers, and down to generation and storage layers). Therefore, this paper encompasses a comprehensive framework to address central smart grids design aspects and suggests a path to integrate the smart grid components into a cohesive and manageable trading platform. Finally, this work demonstrates how the proposed framework can be applied to real market study cases to highlight its solutions, provide a critical evaluation of potential implementation pitfalls, and identify opportunities for further stateof-the-art research.
\end{abstract}

\section{Introduction}

The model used for most of the electricity distribution system which delivers electricity from producers (power plants) to consumers has been operating in the same way for almost a century [1].

This model can be environmentally damaging as it can demand high volumes of fossil fuels and consequently emit particles and gases that contribute to the greenhouse effect. Also, such grids may not be capable to tackle future energy demands [2].

By using new communication technologies, SGs can bring significant contributions to the traditional electric power grid, helping to reduce costs and making operation more flexible and effective. Additionally, SGs can ease Climate Change Mitigation (CCM) and Climate Change Adaptation (CCA) by being capable to integrate renewable energy resources [3] to explore its decentralised energy generation and potential cost reduction.

However, SGs should cover a wide spectrum of demands, for example, user engagement/empowerment [4], renewable energy trading support [5], building renovations [6], optimum utilisation techniques [7] and resiliency (integration, and cyber and physical risks).

SGs are also key components to address the reduction of important greenhouse gases [8] - Carbon Dioxide $(\mathrm{CO} 2)$, Methane $(\mathrm{CH} 4)$ and Nitrous Oxide $(\mathrm{N} 2 \mathrm{O})$ - as described in the United Nations Framework Convention on Climate Change (UNFCCC) and the Paris Agreement. SGs contribute to that by removing fossil fuels from the grid and fomenting the use of renewable energy sources (e.g., Photovoltaic (PV) solar panels and wind power).

To address such demands, SGs should offer solutions for distributed and smart energy management systems. To do so, it is mandatory the development and deploy of innovative solutions and infrastructure backing these systems [9] - especially on what concerns the heterogeneity of smart sensors and other Internet of Things (IoT) devices, and the variety of communication protocols used by them to improve the reliability and security of power grids [10].

The framework presented in this paper offers a highlevel multi-layered view to help in the development of architectural solutions to support business and trading functionalities, communication, and integration layers, as well as generation and storage layers. Through this approach, an overview of the most important aspects of integration and trading are explored.

The rest of this paper is organised as follows: Section 2 reviews related works encompassing important topics for this work. Section 3 offers a high-level multi-layered smart grid framework concerning integration aspects. Finally, Section 4 concludes the paper and suggests future work.

\section{Related Works}

This section surveys state-of-the-art literature about the main topics related to SGs implementation and important bottlenecks/limitations.

As highlighted in [10], IoT technology is at the core of SGs implementations and provides unparalleled 
connectivity at potentially anytime and anywhere. Smart sensors of all sorts, smart meters, and actuators - amongst others - provide the data necessary to monitor and control the SGs and a way to make their operation automatic.

However, the author also emphasises the problems related to the lack of standardisation, relative low-level of adoption and architecture focusing nearly exclusively on Home Area Networks (HANs) architectures (primarily for remote management and controlling of remote appliances), while neglecting other types of networks such as Neighbourhood Area Networks (NANs) and Wide-area networks (WANs) and systems - generation, transmission and so on.

The work of [11] emphasises another aspect of IoT that be an important challenge for SG implementations: IoT scaling versus the costs concerning a centralised model - especially when its operation relates to the use of blockchain technologies.

This model typically implies in centralised cloud (servers, storage and networking) and complex communication resources that become a serious issue as the number of IoT devices grows to the order of billions of units - not to mention security issues and potential attacks (such as "51\% attack"-like attack [12], denial-ofsleep to drain batteries [13] and physical tampering).

Some of these problems can be addressed by, for example, solutions as Lightweight Scalable Blockchain (LSB) for IoT [14] which proposes a distributed trust method where the processing time to validating new blocks gradually decreases as they build up trust in each other and reduces bandwidth and processing time when compared to the classical blockchains.

One of the most important parts of a SG is the demand response methodology [15] and the demand-side of it plays an essential role in the reduction of peak load - by applying various dynamic pricing schemes, such as Timeof-Use Pricing (TOU), Critical Peak Pricing (CPP), DayAhead Pricing (DAP), and others. With a similar approach, the authors of [16] emphasises the importance of the design and development of a flexible integration system as a way to reduce the cost of energy without necessarily reducing the consumption, but by lowering the consumption during peak hours and, at the same time guaranteeing the consumer empowerment and in control of such choices.

\section{Framework}

The framework proposed by this work, and depicted in Figure 1, describes a high-level layered framework that addresses the most important topics for the establishment of the SG. Its layouts integrate all major components covering from energy generation to trading management into a cohesive platform.

This framework is based on the Cooperative Energy Trading System (CENTS) [17], a collaborative project coordinated by the International Energy Research Centre (IERC) in partnership with industry experts (such as Smart MPower [18] and mSemicon [19]), academic research centres (such as University College Cork (UCC), National University of Ireland Galway (NUIG) and Technological University Dublin (TUD)) and a renewable energy supplier, Community Power [20].

The CENTS project was the only energy related project supported by the Disruptive Technologies Innovation Fund (DTIF) in the first round of the fund. One of the main goals of CENTS is to deliver a blockchainenabled peer-to-peer energy trading platform - in addition to the fundamental hardware requirements and market and regulatory strategies. The framework diagram illustrated in Figure 1 is divided in 8 topics and described in the next subsections. Each subsection uses a reference number associated with specific points depicted in the framework diagram.

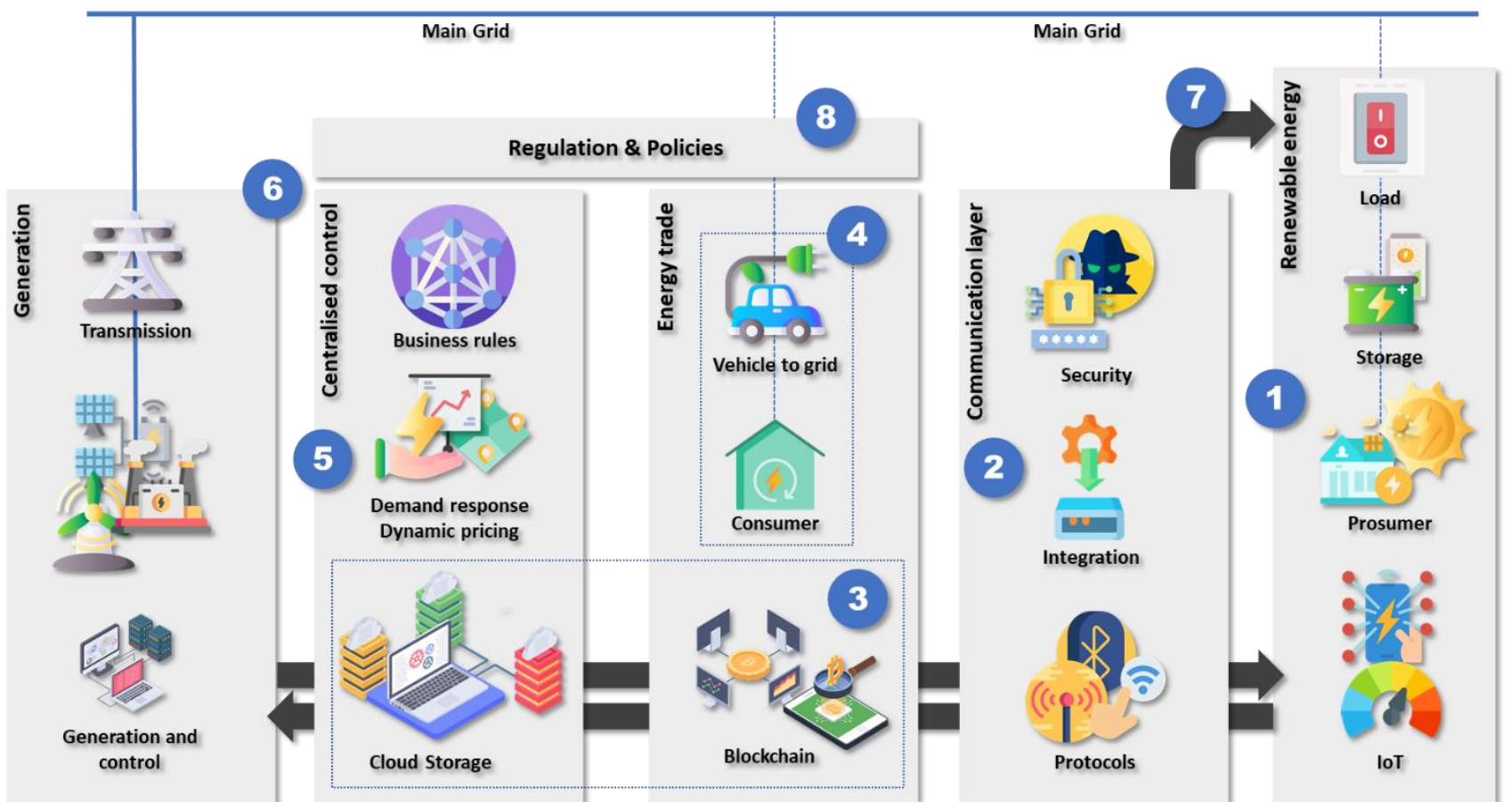

Fig. 1. Framework 


\subsection{Renewable energy}

This layer describes the main components of a distributed renewable energy generation which is mostly focused (or centred) on the consumer empowerment.

\section{Prosumer}

The power grid becomes relatively more complex as the energy generation does not come necessarily only from central power plants. In these scenarios, the individuals can now play more than a "consumer role". As these individuals are now producing energy and feeding it into the power grid, they are referred to as "prosumers" or, in other words, someone that can act as a consumer and/or a producer of energy [21].

\section{IOT}

IoT components (e.g., smart meters, sensors, and actuators) are the core of the SG data generation, monitoring and control. They provide the data needed to make the SG integrated operation viable and, when possible, partial, or completely automatic. However, these IoT components lack standardisation and therefore offer significant challenge when integrating a diverse range of equipment and devices, protocols, and the operation [22].

\section{Storage}

Energy storage is one of the most important components in a grid-connected system. This storage is usually electrochemical (batteries) to make it possible for the prosumer to store the excess energy generated. The energy stored can be traded amongst other prosumers and consumers or even be used for energy maintenance, load shifting, peak shaving and other usages [23].

\section{Load}

The load component is responsible for controlling when and how much energy is consumed from the grid. The energy surplus previously stored can be used and traded amongst consumers or even with Distribution System Operators (DSOs) and in large-scale (such as commercial storage) situations the Transmission System Operators (TSOs). In subsection 7 (Load) there are a few considerations about the implications of the load on the grid.

\subsection{Communication layer}

The communication layer is responsible for integrating and connecting data and functionalities from the distributed renewable energy generators/prosumers and all the other trade participants (e.g., typical energy consumers, regulatory agencies, DSOs/TSOs and so on). Three of the most important components of the communication layer are as follows:

\section{Protocols}

Due to the diversity of hardware and software implementations usually associated with renewable energy generation and IoT devices, the number of distinct protocols in the same site - such as IEEE 802.11 Wi-Fi (Wi-Fi), Nearfield Communication (NFC), Long RangeWide Area Network (LoRaWAN), NarrowBandInternet of Things (NBIoT), Message Queue Telemetry Transport (MQTT), cellular, mesh protocols and others can be a challenge for integration. The lack of standardisation [24], potential interoperability problems and even deleterious mutual interference, can be challenge that would lead to sub-optimal outcomes.

\section{Integration}

The protocol problems show how complex the integration in a SG environment can be. For the scope of this layer and framework, the integration components work as a hub (concentrator) interconnecting all IoT devices and services present in the distributed energy generation sites to the remaining layer of the framework. Specific processes are commonly applied to map and translate the data coming from different devices and protocols. These two-way processes unwrap the data received, convert it to another format, encapsulate it in another format and then transmit it to the proper destination. The standardisation (or the lack of it) is important here and that is exactly what makes the integration components so important to guarantee SG interoperability, compatibility, data integrity, reliability and so on.

\section{Security}

As several IoT devices are connected and controlled in the SGs (e.g., smart meters and transformers), which makes SGs vulnerable to cyber-attacks. These attacks could cause catastrophic damage to these assets, create significant problems for the operation impacting billing, energy balance and even shut an operation down completely.

\subsection{Blockchain}

The use of blockchain in the context of this framework, targets mostly the energy trade aspect of SGs - and not necessarily the IoT data integration. Several solutions have been suggested [25], but they still require fine tuning on both blockchain architecture and the available consensus algorithms to overcome challenges as scalability, power consumption, security, and others. However, blockchain technology has grown in importance to guarantee transactions (e.g., transaction non-repudiation) and is a perfect fit for SGs' trade transactions.

\subsection{Energy trade}

The energy trade layer seeks to deliver blockchain enabled peer-to-peer energy trading functionalities using 
blockchain as its trading transactions record. However, the energy trade relies also on the centralised control layer for it aggregates trade transactions history (customer behaviour of regular consumers, Vehicle-to-Grid (V2G) and so on), dynamic pricing, business rules and even transaction/technical constraints coming from DSOs/TSOs. So, energy trade transactions rely on blockchain to validate the transaction, but the available trade transactions are first validated by the centralised control before they can be concluded $[5,6]$.

\subsection{Centralised control}

A centralised control is still needed to properly address specific (business) rules and/or technical constraints that must consider the relationship with potential consumers and prosumer [5]. For example, the centralised control interacts with DSOs/TSOs to make sure the amount of energy of a transaction is acceptable by the energy grid without risks to the infrastructure and/or customers. This centralised control is intended only to guarantee the compliance with technical constraints and "trade-related" regulations [26]. Other than those, the individual still retains control over its local energy generation and trading. The main topics of a centralised control are:

\section{Business rules}

It is important for a SG to implement specific business rules based on the relationship amongst customers, "seasonal" trading, pricing, technical restrictions, and all sorts of other trading rules (e.g., transaction fulfilment, surplus capacity, and other checks).

\section{Demand response/Dynamic pricing}

The demand response is responsible for developing the support to generation and distribution of renewable energy sources in what concerns an ideally bi-directional automation system [27]. Additionally, it is here where dynamic price algorithms can work - in conjunction with DSOs/TSOs and other trading partners - to define best mechanisms for energy trade (e.g., "seasonal" prices, auctions, reverse auctions and so on).

\section{Cloud storage}

The core of the centralised control, the cloud storage targets high availability and scalability to ensure the business continuity and real-time trading [1]. Also, the centralised control layer keeps track of customer behaviour to support the business rules. Additionally, the data about the transactions is used in the forecasting of energy generation and it the basis for the definition of dynamic pricing and demands and response capabilities.

\subsection{Generation}

Although this layer typically works in an "old-grid" style (centralised generation and control), when analysed by a SG perspective, it can work more intelligently balancing its own generation and the distributed renewable energy generation [28].

\section{Generation and control}

Both DSOs and/or TSOs can benefit from the relationship with SGs as they allow the DSOs/TSOs to bring the distributed generation into the grid and balance it properly, also acting as regulator for the energy grid integration.

\section{Transmission}

The transmission component of this layer becomes more complex as it must deal with distributed generation and additional technical constraints. Although DSOs/TSOs can use the new energy generation available and therefore make the energy distribution more dynamic, the DSOs/TSOs have also to be able to respond quickly to unforeseen conditions (such as weather conditions impacting the predicted PV energy generation, prosumer misbehaviour and so on).

\subsection{Load}

This specific component is an illustration as an external energy trade or technical constraints regulator - such as the DSOs/TSOs - can take control on how much and when the generated renewable energy can be added (or not) to the grid. These functionalities are essential to guarantee safe operation and grid stability.

\subsection{Regulation \& policies}

Like the problem faced with the lack of standardisation, the lack of specific policies and/or regulation for SGs can have huge impact in operation, validation and the relationship with all stakeholders (consumers, blockchain, DSOs/TSOs and government. However, due to the importance given to the decarbonisation challenges all over the world, the discussions and works promoted by academia, private sector and governments are in full swing.

\section{Implementation and usage}

The framework proposed in this research is an integral part of the CENTS [17] project. Through a consortium of research experts, the CENTS project is implementing this multi-layered framework to decouple the main components of a typical SGs' components.

Different consortium' participants focus in the implementation of isolated parts of the proposed framework. For example, while a partner focus on the development of specific smart IoT devices and actuator (module 1 in Figure 1), other partners work on the blockchain backbone to support energy trade (modules 3 and 4 in Figure 1) or support the development of new regulations and policies in close interaction with energy regulatory bodies and/or standardisation groups. 
The integration and protocol definition of these components are in course and so are the simulations and operational tests. The main concern in this step is how the interaction of renewable energy generation (load) and the grid (modules 6 and 7 in Figure 1) would impact the grid or, more specifically, the local grid/substations directly connected to the renewable energy prosumer.

Finally, the simulations and operational tests will bring also important contribution to the standardisation efforts. In addition, this research also brings significant contributions to the development of regulations and policies to foment the adoption of SGs, engage the community and empower the user.

\section{Conclusions}

The use of a multi-layered framework proposed in this work provides a decoupled architecture that allows faster adaption to change. Given that the status of standardisation, regulation and policies are in constant movement (due to the relatively new issues brought by the dynamic characteristics of SGs), this "layered approach" permits a faster adaption to change and significant lower impact to the SG as a whole.

Future works should explore in more details how to adapt promptly to potential significant changes in regulation and public policies. This type of change can bring significant impact over the SG's operation and, to guarantee a smooth transition/adaptation, should such situations demand it. Also, the renewable energy trading and potential high number of prosumers brings a more complex scenario in what concerts pricing and demand forecast, and as such, will demand additional research.

The authors would like to acknowledge the support of Department of Business, Enterprise and Innovation, via its Disruptive Technologies Innovation Fund (DTIF) [29] which provided funding for the CENTS project under the Government of Ireland's Project 2040 Plan [30].

\section{References}

1. G. Dileep, Renewable Energy 146, 2589 (2020)

2. E. Espe, V. Potdar, E. Chang, Energies 11, 2528 (2018)

3. B. Atanasiu, C. Despret, M. Economidou, J. Maio, I. Nolte, O. Rapf, J. Laustsen, P. Ruyssevelt, D. Staniaszek, D. Strong et al., Europe's buildings under the microscope (2011)

4. B. Massey, P. Verma, M.S.K. Khadem, Citizen Engagement as a Business Model for Smart Energy Communities, in Environment-Friendly Energies and Applications (EFEA), 2018 5th International Symposium (Rome, Italy, 2018), p. 6

5. P. Verma, B. O'Regan, B. Hayes, S. Thakur, J.G. Breslin, Energy Inform 1, 14 (2018)

6. B. O'Regan, E. O'Leidhin, F. Tahir, K. Mould, S. O'Donovan, S. O'Sullivan, A. Hryshchenko, D. O’Sullivan, Proceedings 65, 32 (2021)
7. M. Suresh, R. Meenakumari, Transactions of the Institute of Measurement and Control 43, 21 (2021)

8. EU, Clean energy for all Europeans. (2019)

9. V. Caballero, D. Vernet, A. Zaballos, G. Corral, Sensors 18, 400 (2018)

10. Y. Saleem, N. Crespi, M.H. Rehmani, R. Copeland, IEEE Access 7, 62962 (2019)

11. S. Moin, A. Karim, Z. Safdar, K. Safdar, E. Ahmed, M. Imran, Future Generation Computer Systems 100, 325 (2019)

12. D. Bradbury, Computer Fraud \& Security 2013, 5 (2013)

13. M. Gunasekaran, S. Periakaruppan, Security and Communication Networks 2017, e9863032 (2017)

14. A. Dorri, S.S. Kanhere, R. Jurdak, P. Gauravaram, Journal of Parallel and Distributed Computing 134, 180 (2019)

15. Z. Mahmood, ed., Smart Cities, Computer Communications and Networks (Springer International Publishing, Cham, 2018), ISBN 978-3319-76668-3 978-3-319-76669-0

16. P. Pawar, P. Vittal K, Journal of Energy Storage 25, 100846 (2019)

17. CENTS Project, http://www.centsproject.ie/

18. Smart Tech $\mid$ Alternative Energy Solutions

19. mSemicon Custom Product Development, https://www.msemicon.com/en-GB/

20. Community Power, https://communitypower.ie/ourstory/

21. G. Vieira, J. Zhang, Renewable and Sustainable Energy Reviews 143, 110900 (2021)

22. M. Andoni, V. Robu, D. Flynn, S. Abram, D. Geach, D. Jenkins, P. McCallum, A. Peacock, Renewable and Sustainable Energy Reviews 100, 143 (2019)

23. A.H. Al-Badi, R. Ahshan, N. Hosseinzadeh, R. Ghorbani, E. Hossain, ASI 3, 5 (2020)

24. N. Good, K.A. Ellis, P. Mancarella, Renewable and Sustainable Energy Reviews 72, 57 (2017)

25. S.P. Mohanty, V.P. Yanambaka, E. Kougianos, D. Puthal, arXiv:1909.06496 [cs] (2019), comment: 37 pages, 22 figures, 6 Tables, 1909.06496

26. Y. Sabri, N.E. Kamoun, F. Lakrami, A Survey: Centralized, Decentralized, and Distributed Control Scheme in Smart Grid Systems, in 2019 7th Mediterranean Congress of Telecommunications (CMT) (2019), pp. 1-11

27. I. Alotaibi, M.A. Abido, M. Khalid, A.V. Savkin, Energies 13, 6269 (2020)

28. R. Kempener, P. Komor, A. Hoke, Executive Summary, IRENA (2013)

29. N. Miranda, Disruptive Technologies Innovation Fund (DTIF), https://enterprise.gov.ie/en/What-WeDo/ Innovation-Research-Development/DisruptiveTechnologies-Innovation-Fund/

30. Project Ireland 2040, https://www.gov.ie/en/ campaigns/09022006-project-ireland-2040/ 